\title{
Fake fingerprint liveness detection based on wavelet
}

\author{
Prof. B. G. Warwante ${ }^{1}$, Prof.Dr.Mrs.M.R.Patil ${ }^{2}$, Prof.P.S.Patil ${ }^{3}$, Prof.Sharankumar ${ }^{4}$, Prof.S.S.Mulla ${ }^{5}$ \\ Tatyasaheb Kore Institute of Engineering \& Technology, Warananagar, India ${ }^{1}$ \\ Pricipal, AGMR College of engineering and Technology, Varur $^{2}$ \\ Lecturer, Physics Department, Y.C.Warana Mahavidyalaya,Warananagar ${ }^{3}$ \\ Astt.Prof., Tatyasaheb Kore Institute of Engineering \& Technology, Warananagar ${ }^{4}$ \\ HOD, Electronics Department, SSPM College of Engineering, Kankavali ${ }^{5}$
}

\begin{abstract}
Fingerprint verification is one of the most reliable personal identification methods. It plays a very important role in forensic and civilian applications. In this paper we propose a simple and effective approach for fingerprint liveness detection based on the wavelet analysis of the finger tip surface texture. In present situation, manual fingerprint verification is so tedious, time-consuming, expensive and incapable of meeting today's increasing performance requirements. Wavelet transform which has wide range of applications such as image compression, denoising noisy data, texture classification, etc., is used for fingerprint verification. Experimental results show that our method can successfully differentiate live finger tips from fake finger tips made of most commonly used material in fingerprint spoofing.
\end{abstract}

Keywords: Denoising, Fingerprint, Liveness, spoofing, Wavelet.

\section{INTRODUCTION}

Recent research works [1] have revealed that it is not difficult to spoof an automated fingerprint authentication system (AFAS) using fake finger tips made of commonly available materials such as clay and gelatin. Such fake finger tips can spoof most practical AFASs as long as they can retain some or all of the original finger tips' minutiae information. The process to decide whether a fingerprint collected is really from a live finger tip or not by acquiring and analyzing accessorial finger tip information other the minutiae is called liveness detection. Static properties (e.g. temperature; conductivity) and dynamic behaviors (e.g. skin deformation; perspiration) of live finger tips have been extensively studied in fingerprint liveness detection research [1]. All of the existing approaches are believed to have certain limitations because the properties used are either unstable or not universal enough [1]. This Letter proposes a new fingerprint liveness detection approach based on analyzing a more robust and intrinsic property of finger tips - surface coarseness.

\section{PROPOSED APPROACH}

Popular fake finger tip materials such as clay and gelatin usually consist of big organic molecules which tend to agglomerate during processing. This will introduce asperities to the surface of the fake finger tips produced. So, generally speaking, the surface of a fake finger tip is much coarser than that of a live finger tip (human skin). This difference in surface coarseness will be used in our fingerprint liveness detection method. Nowadays, the resolution of popular fingerprint sensors for public security purposes is around 800dpi. Surface textures are generally not clear enough at such a resolution. Coping with the development of electronic technologies, we can expect that in the near future, relatively low cost fingerprint sensors, especially optical sensors, will be able to generate high resolution (1000dpi) images of finger tips. Here, we propose a liveness detection method based on the analysis of such higher resolution finger tip images. Finger tip surfaces are intrinsically coarse at certain scale because of the alternation of the ridges and valleys on them. Wavelet analysis can help us to minimize the effect of ridge/valley pattern when estimating the surface coarseness because it allows investigation of the input signal at different scales. In the proposed approach, we treat the surface coarseness as a kind of Gaussian white noise added to the images. A finger tip image is first denoised using wavelet based approach [2]. The noise residue (original image - denoised image) is then calculated. Coarser surface texture tends to result in a stronger pixel value fluctuation in the noise residue. Thus, the standard deviation of the noise residue can be used as an indicator to the texture coarseness.

\section{THE APPROACH CONSISTS OF THE FOLLOWING STEPS:}

Step 1: Apply histogram equalization to the input finger tip image.

Step 2: Two levels of stationary wavelet decomposition are performed to the finger tip image. In the second level, only the level one approximation (the down sampled output from the first round of low pass filtering) is further decomposed. Finally, one approximation and six details are achieved. Finite impulse response (FIR) based approximation of the Meyer wavelet is adopted.

Step 3: Wavelet shrinkage is performed by applying softthresholding [3] to the six details. The threshold $\delta$ for the 
wavelet shrinkage is calculated according to equation (1) as proposed in [4]

$$
\delta=2 \log (N) \sigma(1),
$$

Where $\log ()$ stands for natural logarithm; $N$ is the signal length of each detail; $\sigma$ is the estimated standard deviation of the detail coefficients of all the three details in the first level of wavelet decomposition.

Step 4: Perform wavelet reconstruction to get the denoised finger tip image.

Step 5: Noise residue is achieved by calculating the difference between the two finger tip images before and after denoising.

Step 6: If the standard deviation of the noise residue is smaller than a preset threshold, the original image is regarded as been captured from a live finger tip; otherwise, it is regarded as a fake fingerprint.

Fig. 1 compares two 1000dpi finger tip images, one is a human finger tip and the other is a fake finger tip made of plastic clay. Our method is applied to these two images and the difference between the two values of noise residue standard deviation is obvious.

\section{EXPERIMENTAL SETUP}

A high resolution ( 1000dpi) finger tip image database was created. Due to the lack of suitable fingerprint sensors, a Fuji S2 pro 12 million-pixel Digital Single Lens Reflex (DSLR) camera was used to capture the images. The database contains images of 23 real finger tips; 10 gelatin fake finger tips and 24 plastic clay fake finger tips [5]. 200 sub-images of size $256 \times 256(0.65 \mathrm{~cm} \times 0.65 \mathrm{~cm})$ were randomly cropped from the high resolution finger tip images in the database.

Among them, 100 sub-images are from real finger tip images; 50 are from gelatin finger tip images and the rest 50 are from plastic clay finger tip images.

The proposed algorithm was applied to all the 200 subimages to get the standard deviations of their noise residues. Matching results shown in Fig. 2 exhibit that our method was able to successfully differentiates all the fake finger tips from the real ones.

Our method is also quite efficient (real time) as only two levels of wavelet analysis and a small part of finger tip image are needed.

To facilitate fingerprint liveness detection research, we also collected fingerprints of the fake finger tips described above as well as their corresponding live finger tips using several sate-of-the-art fingerprint sensors. The databases are available for download [5] by research community. The procedures for making these fake finger tips can also be found at [5].

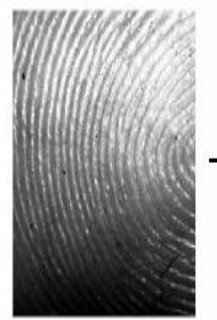

Live finger tip

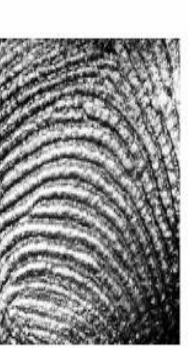

Fake finger tip

made of plastic clay

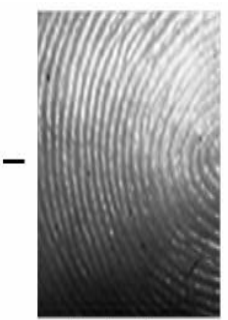

Denoised image using Dmey wavelet

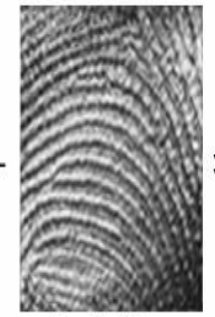

Denoised image using Dmey wavelet

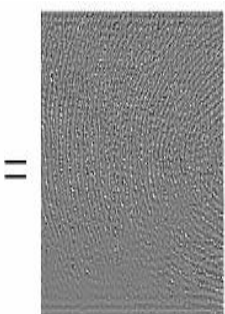

Noise residue Standar Deviation $=11.5$

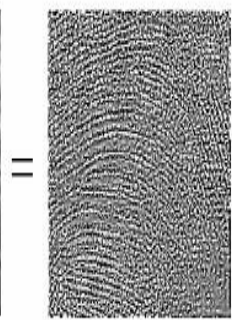

Noise residue

Standar Deviation $=36.5$
Fig. 1 Fingerprint liveness detection using wavelet based denoising

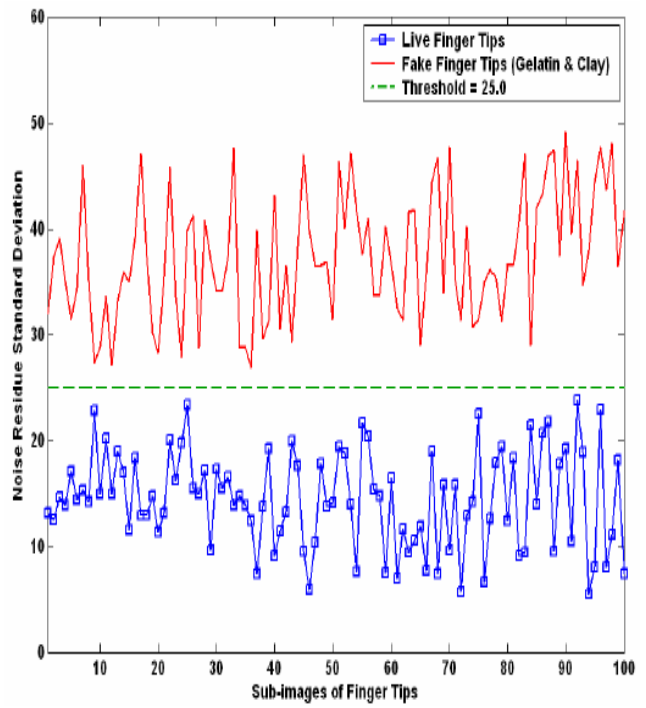

Fig. 2 Experimental results of the proposed method

\section{CONCLUSION}

A new forward-looking fingerprint liveness detection method has been proposed. Experimental results demonstrate its effectiveness on high resolution finger tip images which can probably be generated by next generation higher resolution fingerprint sensors. The method is efficient enough so that its incorporation into existing fingerprint verification systems will not downgrade the real time performance of such systems in public security applications.

\section{REFERENCES}

[1] D. Maltoni, D. Maio, A. K. Jain, and S. Prabhakar. Handbook of Fingerprint Recognition, Springer Verlag, New York, USA, June 2003, pp. 286-291

[2] W. S. Lu, Wavelet Approaches to Still Image Denoising, Conference Record of the 31st Asilomar Conference on Signals, Systems \& Computers, vol. 2, Nov. 1997, pp. 1705-1709

[3] D. L. Donoho, De-Noising by Soft-Thresholding, IEEE Transactions on Information Theory, vol. 41, Issue 3, May 1995, pp. 613 - 627

[4] D. L. Donoho, I. M. Johnstone, Ideal Spatial Adaptation by wavelet shrinkage, Biometrika, vol. 88, 1994, pp. 425-455

[5] http://fpserver.cse.cuhk.edu.hk/liveness.htm 